\title{
THROMBOEMBOLIC EVENTS IN SYSTEMIC LUPUS ERYTHEMATOSUS IN AMAZONAS
}

Andrezza Mendes Franco ${ }^{1, \star}$, Camylla Rita Lima do Nascimento ${ }^{1}$, Vitor Matheus Orlando Sampaio1, Camile Smith de Oliveira Brito $^{1}$, Ingrid dos Santos Araújo ${ }^{1}$, Sebastião Barreto Falcão Neto ${ }^{1}$, Clara Pinheiro Martins ${ }^{1}$, Igor Oliveira da Silva ${ }^{1}$, Sandra Lúcia Euzébio Ribeiro ${ }^{1}$

1.Universidade Federal do Amazonas, Manaus (AM), Brazil.

*Corresponding author: andrezzamf2190@gmail.com

\section{BACKGROUND}

Systemic lupus erythematosus (SLE) is a chronic and multisystemic inflammatory disease characterized by the presence of several autoantibodies. It is a disease that predominates in females and African Americans. Clinical manifestations can be from cutaneous and articular involvement to the occurrence of neurological events and cardiovascular graves. The aim of this study is to analyze the prevalence of SLE thromboembolic events in the state of Amazonas.

\section{MATERIALS AND METHODS}

A retrospective, observational, cross-sectional and descriptive study that analyzed a sample of 200 medical records of patients with SLE of both sexes and all age groups, in regular follow-up at a reference service in rheumatology in Amazonas. Sociodemographic data, occurrence of thrombosis and details of these events were displayed.

\section{RESULTS}

The mean age of the patients included in the study was $43.4 \pm 11.9$ years, the majority being female (91\%) and nonwhite ( $81.2 \%)$. It was found that $20 \%$ had a thrombosis record, the main one being arterial (55\%), followed by venous $(47.5 \%)$ and microvascular (20\%) thrombosis. Within this group, the main events that occurred were deep venous thrombosis, stroke, acute myocardial infarction and transient ischemic attack. In addition, 24 patients with a diagnosis of antiphospholipid antibody syndrome (APS) were found, a factor that led to higher rates of thromboembolic events.

\section{CONCLUSION}

It is observed that the prevalence of thromboembolic phenomena in patients with SLE studied is higher than the general population, especially the arterial type, a fact that is justified in part by the higher cardiovascular risk that SLE causes. In contrast, the coexistence of APS in some patients agrees with the existence of an important prothrombotic condition, as reported in the literature. Therefore, it is necessary to employ more care and attention in the clinical management of patients, training in the prevention and early treatment of potentially serious thrombotic conditions. 